\title{
SUPERHUB: Integrating behaviour change theories into a sustainable urban-mobility platform
}

\author{
Paula J. Forbes \\ paula.forbes@abdn.ac.uk
}

\author{
Simon Wells \\ simon.wells@abdn.ac.uk \\ Computing Science, University of Aberdeen, Aberdeen, AB24 3UE \\ Judith Masthoff \\ j.masthoff@abdn.ac.uk
}

\author{
Hien Nguyen \\ n.nguyen@abdn.ac.uk
}

\begin{abstract}
The SUPERHUB project (SUstainable and PERsuasive Human Users moBility in future cities) promotes the creation of a new urban mobility services ecosystem to facilitate the take-up of environmentally sustainable behaviours. It will design and test an open platform able to combine in real time all mobility offers from the relevant stakeholders together with a set of enabling mobility services able to address users' mobility needs and to foster behavioural change. This paper explores how SUPERHUB plans to integrate behaviour change theories.
\end{abstract}

Keywords. Behaviour Change, Greenhouse Gas Reduction, Sustainable Travel, Persuasion, Transport, Urban Mobility.

\section{INTRODUCTION}

World population is increasingly city based with $51 \%$ or 3.5 billion people living in urban areas. Existing mobility systems are under strain and are using increasing amounts of resources. The transport sector represents $30 \%$ of the final energy consumption of the EU and is a major source of greenhouse gas emissions and pollutants. If we are to ensure sustainable development for Europe as well as meet EU targets for greenhouse gas emissions and energy efficiency it is vital that we have an efficient and usable mobility system that has as little impact on the environment as possible. SUPERHUB aims to provide an integrated, multimodal system that supports transport needs and encourages environmentally sustainable choices to help limit the environmental impact of transport. It is a collaboration of 20 European partners, including academic institutions, transport companies, environmental agencies and city councils. (See superhub-project.eu for a full list and more detail). Another aim is to investigate what are the most effective methods to persuade people to adopt environmentally sustainable behaviour. To achieve behaviour change, one option is to lower the barrier for uptake of a behaviour, so a system that makes it easier for people to find mobility information, book public transport and share lifts may encourage sustainable behaviour. Therefore, SUPERHUB aims to empower people with information, providing accurate, real time data on travel options and any disruptions to services, and providing support for car pooling and taxi sharing. Crowd sourcing will be used to provide up-to-date information (e.g., on transport delays and the crowdedness of busses), whilst care will be taken to ensure the reliability of information. Additionally, behaviour change can be achieved by employing more direct behaviour change techniques. SUPERHUB will therefore support the setting of individual mobility related goals (e.g. use public transport or provide a lift at least once a week), and provide feedback on mobility behaviour and encouragement to do better. SUPERHUB will also exploit the potential of serious games and social networks to sensitize people and make them reflect about their environmental behaviour. This paper is mainly concerned with the second way of achieving behaviour change, and explores how to integrate behaviour change theories into SUPERHUB.

\section{HOW CAN WE PERSUADE PEOPLE TO MAKE SUSTAINABLE CHOICES?}

There is a section of society that is committed to the environment and will do what they can to reduce their carbon footprint. Others may take more persuading to make more sustainable travel choices. Anable (2005) states that travel research methodology and policy interventions often overlook how the combination of instrumental, situational and psychological factors affect travel choice and how these differ for distinct groups of people. Understanding what will motivate people to change their behaviour is a key element of successful persuasion. For example, visualising the amount of $\mathrm{CO} 2$ produced over a year may work for some whereas for others finding out the amount of money they could save by taking the bus rather than driving may be more motivating (as found in our focus groups). Different people will respond more or less to different cues and we aim to investigate what the most effective methods for persuasion are and how they can be implemented. We want to provide a personalized system that optimally persuades people to make more environmentally aware transport choices. 


\section{PERSUASIVE TECHNOLOGY}

Persuasive technology is technology specifically designed to change people's attitudes and/or behaviours (Fogg, 2003). Persuasion implies a voluntary change, without using coercion. Persuasive systems aimed at changing behaviour are often called "Digital behaviour interventions".

\subsection{Theories of behaviour change}

There is growing evidence that using behaviour change theory leads to more effective interventions. For example, a systematic review of 85 studies involving 43,236 participants found that "the effectiveness of Internet-based interventions is associated with more extensive use of theory (in particular the use of the theory of planned behaviour)" (Webb, 2010). Many theories exist; see Jackson (2005) for an overview. These theories help to identify the key behavioural determinants (Michie et al, 2005). These determinants are then targeted by behaviour-change techniques. Advantages of the theory-based approach include: (1) interventions are likely to be more effective if they target causal determinants of behaviour and behaviour change; this requires understanding these causal determinants, i.e. theoretical mechanisms of change; (2) theory-based interventions facilitate an understanding of what works and thus a basis for developing better theory across different contexts, population and behaviours; (3) vice versa, theories can be tested and developed by evaluations of interventions only if those interventions and evaluations are theoretically informed. Fogg (2003) proposed an integrated model within the field of persuasive technology. Aiming at intervention designers who need an easy-to-use practical framework, Fogg's behaviour model is deliberately simple: it states that three elements -namely motivation, ability and a trigger- must occur at the same time for behaviour to happen. The lack of any of these three elements will cause non-compliance. The Fogg model says that if you get the motivators right, and if the behaviour is made easier for people to do, and if you trigger it - then the behaviour is more likely to occur. Considering the many theories, some attempts, based on expert consensus, have been carried out to identify a set of common behaviour determinants. Fishbein et al (2001) analysed behaviour change theories to change people from risky to healthy HIV preventive behaviours. They identified eights factors that enable predicting and understanding behaviour. Michie et al (2005) analysed 33 theories with 128 constructs (including the 5 used by Fishbein et al) from a wide variety of fields, and identified 12 factors that are most likely to influence behaviour.

\subsection{Behaviour change techniques}

Behaviour change techniques are strategies used to promote behaviour change (Webb, 2010). For example, an intervention designed to encourage people to walk more can ask them to monitor their daily step count to raise their awareness of their current behaviour - the 'self-monitoring' technique. Each theory of behaviour change is associated with a number of techniques, each of which can be further mapped to a specific behavioural predictor defined in the theory. Many researchers have attempted to create a taxonomy of behaviour change techniques. For example, the taxonomy developed by Michie et al. (2008) has defined and mapped 137 techniques to 11 behavioural predictors with the indication of where they can be used effectively. Behaviour change theories can be used to predict which combinations of techniques are likely to be most effective. For instance, Control Theory suggests how feedback may interact with other techniques to change behaviour. In the domain of healthy eating and physical activity interventions, Michie et al's (2009) meta-analysis of 122 studies found that interventions that combined self-monitoring with at least one other "selfregulatory" technique were twice as effective when compared with other interventions. Based on their analysis, they suggested the inclusion of five techniques: prompting intention formation, prompting specific goal setting, providing feedback on performance, prompting self-monitoring of behaviour, and prompting review of goals.

\subsection{Personalising the intervention}

Theories of behaviour change agree that any voluntary change of behaviour is not an event, but a process (e.g. Prochaska \& Norcross, 2001). This process can go from not wanting to change, to considering change, to making and maintaining permanent change. Such a process develops over a long time. This is particularly true when the problematic behaviour is an everyday habit (e.g. travelling by car). People neither go through the process of change in the same order nor at the same speed. Additionally, behavioural determinants, the most appropriate behaviour change techniques and optimal mode of delivery may depend on the user. Any behaviour intervention, therefore, must be tailored to the beliefs, preferences, and circumstances of each individual. Empirical evidence supports this view; a meta-analysis by Noar et al. (2007) showed tailored messages outperformed comparison messages in affecting health behaviour change.

\section{EXISTING PERSUASIVE TECHNOLOGY FOR SUSTAINABLE TRANSPORT}

Although the majority of research into persuasive technology has been in the health domain, there are examples of persuasive technology research in the transport domain. A particular focus has been 
on 'mobility management' systems that motivate people to use more sustainable forms of transport by providing detailed travel information, incentives for selecting more sustainable modes of transport, and applying marketing techniques which focus on individual travel behaviours (Jones, 2003; Taniguchi et. al. 2007). For EU countries, the European Platform on Mobility Management (EPOMM) and the European Local Transport Information Service (ELTIS) provide a large number of case studies about implementing mobility management measures. In the transport domain, the dominant form of digital behaviour intervention is the 'travel feedback program' which gives feedback on $\mathrm{CO}_{2}$ emissions estimates, advice on car use reduction, information on public transport, etc. Examples include Travel Blending (Rose \& Ampt, 2001), TravelSmart (Ampt \& Rooney, 1999), Wise Ways to Use Cars (Taniguchi et al 2003), and the UK personalized travel planning systems reported in the Smarter Choices document (Cairns et al, 2004). To give an indication of the effectiveness of these approaches, a meta-analysis in Japan by Taniguchi et. al. (2007) of travel feedback programs found a mean reduction in car use of $19 \%$, while the Cairns et al. (2004) analysis in the UK reported a $7-15 \%$ reduction in car use in urban areas.

\section{DESIGN AND EVALUATION OF SUPERHUB}

To ensure that SUPERHUB provides an effective tool, it will be developed in a User-Centric way, putting the user at the heart of the design process. Our target users will be anyone who travels and could use more environmentally sustainable modes of transport than they currently do, even for some of the journeys that are made. We also aim to support those already using sustainable transport. Requirements gathering research has already taken place in SUPERHUB's three trial cities: Barcelona, Helsinki and Milan. Quantitative data was obtained by questionnaire (about 200 responses per city) and qualitative data by running Focus Groups (about 10 focus groups in each city, using different kinds of transport users). Scenarios were developed to showcase the core SUPERHUB functionalities, relevant scenarios were then discussed in the Focus Groups. This initial research provided a large amount of data on demographics, current transport usage, problems experienced, mobility preferences as well as feedback and inspiration for new scenarios. The wide range of attitudinal data will ensure that we are as well informed of the needs of the target users as possible. Full details of this preliminary work can be found in the Superhub deliverable D1.1 (www.superhub-project.eu). Data is still undergoing analysis but we intend to carry out factor analysis and then segmentation analysis to identify homogenous groups or clusters of cases.
We are currently running a series of Participatory Design workshops with a variety of end-users who will play a key role in the development of the system. In each city, the SUPERHUB functionalities will then be tested in a realistic environment with more than 200 users, investigating the impact of SUPERHUB solutions in different contextual backgrounds. We are also developing Key Performance Indicators (KPI's) to measure how successful aspects of the system are. KPl's would include measurements of long term user adoption, user satisfaction, attitude change and knowledge of environmental issues.

\section{PERSUASIVE TECHNOLOGY IN SUPERHUB}

SUPERHUB plans to go beyond the state of the art for persuasive systems within the sustainable transport domain in two main ways. Firstly, it will work with users through participatory design and user-centred development to build a system in which the functionality focuses on the real-world transport needs of the user. Secondly, it will develop a system that is able to facilitate desirable behavioural shifts by utilising much more of the potential of persuasive technologies. SUPERHUB will facilitate behavioural shifts using a subset of the techniques identified by Michie et al (2008). The following techniques (which include the five found most effective by Michie et al., 2009) will form the core of SUPERHUB's intervention:

- Prompting intention formation, specific goal setting and goal review. Users set specific mobility related goals which are reviewed regularly to determine to what extent the goal has been met and whether to adjust it.

- Monitoring, feedback, and rewards. Participants' travel behaviour is monitored, feedback on behaviour is provided, and rewards are provided if appropriate. The rewards to use (e.g. reduced bus fares, social recognition) will be determined during participatory design.

- (Social) Comparison. Comparative data shows participants how their behaviour compares with their past behaviour, and that of others in the community or support group.

- Prompts and personalisation. Participants are prompted at appropriate times to change their behaviour, e.g., to provide a lift or use public transport. Prompts are personalised to participants' characteristics and contextual circumstances. For example, the focus groups showed that adaptation is needed to the weather and travel company.

- Aiding decision-making. Users will be provided with sufficient appropriate information to enable them to make informed decisions about their travel behaviour. 
SUPERHUB will use information push via mobile devices to prompt users about their transport decisions and to make suggestions about alternatives. For example, when a user has indicated that they are willing, in principle, to take part in car-pooling, SMS can be used to organise an impromptu car-pool in real-time rather than relying on pre-arranged, longer-term planning. Decisions about how and when to make these kinds of interventions will be based upon datamining of users' profiles and travel-behaviour metrics and use contextual information such as adverse weather. Furthermore, data mining enables alternative behaviours to be suggested. For example, when the system has learnt about a user's regular travel habits, suggestions can be made such as to try public transport for a given leg of their journey. In this way the persuasive elements of the systems seamlessly integrate into the travel planning functionality. Data-mining will also be used to inform the persuasive functionality in profile matching, users whose profiles suggest compatibility may be matched up for a car-pooling or taxi sharing offer if their travel plans coincide. We aim to create a synthesis of automated digital interventions based on intelligent analysis of tracked user behaviour together with explicit goal setting, adjustment, achievement-tracking, feedback and incentive mechanisms. Automated interventions will cover un-prompted, opportunistic contextual interventions, such as suggesting alternatives, like renting a bike when a scheduled bus is late, or utilising social compatibility matching to formulate persuasive messages encouraging a user to join a car pool with someone with whom they have similar hobbies, as well as the supportive role found in conscious goal-setting based approaches to behaviour management.

\section{FUTURE WORK}

We are currently running participatory design workshops to inspire the user interface and the persuasive component. We will perform foundational research into mobility behaviour and expectations, with special attention to sustainability and motivational aspects; create mock-ups and prototypes to be used in formative and summative evaluations. Testing will be scaled up later to include around 1000 people per trial city. Further details can be found at: superhub-project.eu

\section{REFERENCES}

Ampt, E., and Rooney, A. (1999) Reducing the Impact of the car: A sustainable approach. Travel Smart Adelaide, Perth, Australia

Anable, J. (2005) 'Complacent Car Addicts' or 'Aspiring Environmentalists'? Identifying travel behaviour segments using attitude theory. Transport Policy 12, 65-78.

Cairns, S. et al. (2004) Smarter choices: Changing the way we travel, Department for Transport, UK.

European Local Transport Information Service (2012). eltis.org (retrieved 14.06.2012)

European Platform On Mobility Management (2012) epomm.eu (retrieved 14.06.2012)

Fishbein, M. et al. (2001). Factors influencing behaviour \& behaviour change. Handbook of health psychology. Mahwah,NJ: Lawrence Erlbaum, 3-17.

Fogg, B.J. (2003). Persuasive technology: using computers to change what we think and do, Morgan Kaufmann

Jackson, T. (2005). Motivating sustainable consumption: A review of models of consumer behaviour and behavioural change. London: Policy Studies Institute.

Jones, P. (2003). Encouraging behavioural change through marketing and management: What can be achieved? Conf. Travel Behaviour Research.

Michie, S. et al. (2005). Making psychological theory useful for implementing evidence based practice: a consensus approach. Quality and Safety in Health Care, 14, 26-33.

Michie, S. et al. (2008). From theory to intervention: mapping theoretically derived behavioural determinants to behaviour change techniques. Applied Psychology, 57 (4). 660-680

Michie S. et al. (2009). Effective techniques in healthy eating and physical activity interventions: a meta-regression. Health Psych., 28(6), 690-701

Noar, S.M., Benac, C.N. and Harris, M.S. (2007). Does tailoring matter? Meta-analytic review of tailored print health behaviour change interventions. Psych. Bulletin, 133(4), 673-693

Prochaska, J. O, Norcross, J. C. (2001). Stages of change. Psychotherapy, 38, 443-448

Rose, G., and Ampt, E. (2001) Travel blending: an Australian travel awareness initiative. Transportation Research, 6, 95-110.

Taniguchi, A. et al. (2003) Psychological and behavioural effects of Travel Feedback Program for travel behaviour modification, Transportation Research Record, 1839, 182-190.

Taniguchi, A., Suzuki, H., Fujii, S.(2007). Mobility management in Japan: Its development and meta-analysis of travel feedback programs. Transportation research record, 2021, 100-109.

Webb, T.L., et al. (2010). Using the internet to promote health behaviour change: A systematic review and meta-analysis. Journal of Medical Internet Research 12(1) 